\title{
Optical and near-infrared simultaneous observations of the BL Lacs PKS 2005-489 and PKS 2155-304^
}

\author{
T. P. Dominici, Z. Abraham, and A. L. Galo
}

\author{
Instituto de Astronomia, Geofísica e Ciências Atmosféricas, Universidade de São Paulo, Rua do Matão 1226, Cidade Universitária, \\ 05508-900, São Paulo, SP, Brazil \\ e-mail: [tania;zulema;galo]@astro.iag.usp.br
}

Received 5 May 2005 / Accepted 3 August 2006

ABSTRACT

\begin{abstract}
Aims. In this work, we looked for simultaneous brigthness variations in the optical and near-infrared bands in the TeV BL Lacs PKS 2005-489 and PKS 2155-304, covering time scales from minutes to a few years.

Methods. Between 2001 and 2003, we carried out optical and near-infrared observations using two telescopes at the Pico dos Dias Observatory (LNA, Brazil), reaching a time resolution in the light curves of about 14 min at optical wavelengths and 6 min in the infrared. For both sources the variability analysis was done after the contribution of the host galaxy was subtracted from the observed flux.

Results. In PKS 2005-489 we detected for the first time optical and near-infrared variations on time scales of minutes. Both sources presented variability on different time scales and with complex and distinct spectral behavior. This behavior favors a scenario in which the variability is produced by the formation and fast evolution of shock waves in the quiescent or slowly varying nuclear jet.
\end{abstract}

Key words. galaxies: BL Lacertae objects: individual: PKS 2005-489 - galaxies: BL Lacertae objects: individual: PKS 2155-304

\section{Introduction}

In active galactic nuclei (AGNs), the variability on short time scales is more common in the class of blazars, which includes the BL Lacs, optical violent variable objects (OVVs), and flat spectrum radio Quasars (FSRQ). Variability puts constraints on the size of the emitting region and, in general, relativistic effects must be considered to conciliate the inferred luminosities with the small sizes. Relativistic jets oriented close to the line of sight are very convenient sites for generating the brightness variations in blazars on all time scales, through the formation and propagation of shocks and their interaction with the inhomogeneous surrounding medium (Marscher et al. 1992). A similar scenario, involving the superposition of different components such as the quiescent jet and a shock or evolving knot, was also used to explain the spectral variability of polarized emission in blazars (Brindle et al. 1986; Valtaoja et al. 1991; Andruchow et al. 2003). Geometric effects, such as the change in the angle between the jet and the line of sight, can explain symmetric flares (Wagner et al. 1995) and periodic components in the light curves (e.g. OJ 287, Abraham 2000).

For some sources, the Lorentz factor of the relativistic bulk motions needed to explain rapid variability are much higher than those derived from the kinematics of the jet components (e.g. Qian et al. 1991). Models of extrinsic processes, such as interstellar scattering or microlensing, have been proposed to solve this discrepancy (Romero et al. 1995; Wagner \& Witzel 1995; Kraus et al. 1999). While the first process has observable effects only at radio wavelengths, microlensing models predict achromatic variability at all wavelengths. Many campaigns were undertaken to detect optical short-term variability in blazars (e.g.

^ Based on observations made at the Laboratório Nacional de Astrofísica (LNA-CNPq, Brazil).
Heidt \& Wagner 1996; Jang \& Miller 1995, 1997; Romero et al. $1999,2002)$, although most of them were restricted to only one spectral energy. For that reason, a pattern of rapid variability behavior across the eletromagnetic spectrum and the relation among different energy bands could not be established, even for the best-studied sources. Also, little information about the occurrence of rapid variability in blazars in the the near-infrared bands could be found in the literature (Lorenzetti et al. 1989; Whiting et al. 2002).

In recent years, multiwavelength monitoring campaigns have been carried out to focus on a few blazars (e.g. AO $0235+164$, Raiteri et al. 2005; 3C 66A, Böttcher et al. 2005; PKS 2155-304, Urry et al. 1997; Pesce et al. 1997; Pian et al. 1997), in most cases covering frequencies from radio to X-rays. In general, the optical and near-infrared variability seems to be well-correlated and has no measurable delay on any time scale.

It was believed that a common property of all blazars was that the spectrum always becomes flatter when the brightness increases. However, some recent observations in the optical bands have shown that this scenario is not so simple (Ghosh et al. 2000; Gu et al. 2006). Some objects show spectral steepening or no changes when the flux increases in variability events on time scales from months to hours (e.g. S5 0716+71, Raiteri et al. 2003; AO 0235+164, Ghosh et al. 2000; BL Lac, PKS 0736+017, Clements et al. 2003). These new data could be indicating that we are observing a superposition of components with different spectral and temporal properties. In particular, Gu et al. (2006) suggest that the steepening of the spectrum during the flux increase is more common in blazars classified as flat spectrum radio quasars (FSRQ), which can be due to the presence of a more significant thermal component. More observations at optical and near-infrared wavelengths are needed to investigate the relationship between variability and the spectral behavior on different time scales. 
Optical observations have shown that microvariability is more common in radio-loud sources; a useful number for quantifying the incidence of rapid variability is the duty cycle, which represents the fraction of the time that a source (or a class of sources) varies on a given time scale. Particularly, the optical microvariability duty cycle for radio-loud and radio-quiet objects calculated by Romero et al. (1999) was 68\% and 6.9\%, respectively, while for the specific case of X-ray selected BL Lacs (XBLs), it was $27.9 \%$, and $67 \%$ for radio-selected BL Lacs (RBLs).

In 2001 we started an observational project that consisted of optical and near-infrared simultaneous observations of blazars with high temporal resolution. In this work we present the results for the BL Lac objects PKS 2005-489 and PKS 2155-304, for which optical light curves covering five years of observations were recently presented (Dominici et al. 2004).

PKS 2005-489 ( $V=13.4, z=0.071$, Véron-Cetty \& Véron 1998 ) is considered an RBL, although its synchrotron peak is located between the UV and X-rays. Variations in the $R$ band, on time scales of a few days, have already been observed in 1990 by Heidt $\&$ Wagner (1996) and in the $B, V$, and $R$ bands by Rector \& Perlman (2003) during August 2000, the latter apparently without any wavelength dependence. Dominici et al. (2004) found variations of about $1 \mathrm{mag}$ in the $V$ band on time scales of about 20 days and a possible correlation with the X-ray light curves from the All Sky Monitor (RXTE/ASM). However, variability on shorter time scales was found neither during the 1997 observations of Romero et al. (1999), in the $V$ and $R$ bands, nor in August 2000 (Rector \& Perlman 2003). Recently, this object was also observed at energies higher than $200 \mathrm{GeV}$ by the HESS telescopes (Aharonian et al. 2005b), and is the second southern-hemisphere blazar detected at these energies.

The XBL PKS 2155-304 ( $V=13.1, z=0.116$, Véron-Cetty \& Véron 1998) presents optical variability on time scales of months, days, and minutes (Edelson et al. 1995; Courvosier et al. 1995; Pesce et al. 1997; Urry et al. 1997; Pian et al. 1997; Paltani et al. 1997; Dominici et al. 2004). However, a search for optical microvariability $(V)$ in 1997 and 1998 carried out by Romero et al. (1999) gave negative results, confirming that for this source short-term variability occurs in isolated epochs (Heidt et al. 1997). It was the first southern-hemisphere blazar detected at TeV energies (Chadwick et al. 1999; Aharonian et al. 2005a).

In this paper we present probably the first detection of variability in PKS 2005-489 on time scales of minutes. We also detected short-time variability in PKS 2155-304 and confirmed the duty cycle of about $30 \%$ found by Romero et al. (1999). In addition, we present long-term infrared observations and a study of the optical and near-infrared spectral behavior on different time scales for both sources. In Sect. 2 we describe the observational program and data reduction procedures. The resulting differential light curves are discussed in Sect. 3. The spectral behavior of the sources during the monitoring program is presented in Sect. 4. The calculation of the duty cycle is shown in Sect. 5. Finally, in the last section we summarize the results.

\section{The observational program}

The observations were carried out in two campaigns of three nights each, in July and August of 2001. PKS 2155-304 was also observed one night at optical wavelengths and two nights in the infrared in November of that year. Two telescopes at the Pico
Table 1. Log of the observations for PKS 2005-489.

\begin{tabular}{|c|c|c|c|c|c|c|c|}
\hline Date & Filter & $N$ & microvar? & Date & Filter & $N$ & microvar? \\
\hline \multirow[t]{6}{*}{$07 / 16 / 01$} & $B$ & 18 & yes & $08 / 12 / 01$ & $B$ & 4 & no \\
\hline & V & 19 & yes & & V & 10 & no \\
\hline & $R$ & 19 & yes & & $R$ & 10 & no \\
\hline & $I$ & 13 & yes & & $I$ & 3 & no \\
\hline & $J$ & 24 & yes & & $J$ & 10 & no \\
\hline & $H$ & 22 & yes & & $H$ & 7 & no \\
\hline \multirow[t]{6}{*}{$07 / 17 / 01$} & $B$ & 13 & yes & $07 / 08 / 02$ & $J$ & 9 & no \\
\hline & V & 22 & yes & & $H$ & 8 & no \\
\hline & $R$ & 22 & yes & & & & \\
\hline & $I$ & 12 & yes & & & & \\
\hline & $J$ & 25 & yes & & & & \\
\hline & $H$ & 25 & yes & & & & \\
\hline \multirow[t]{6}{*}{$07 / 18 / 01$} & $\bar{B}$ & 10 & no & $07 / 09 / 02$ & $J$ & 2 & no \\
\hline & V & 20 & no & & $H$ & 2 & no \\
\hline & $R$ & 20 & no & & & & \\
\hline & $I$ & 10 & no & & & & \\
\hline & $J$ & 26 & no & & & & \\
\hline & $H$ & 25 & no & & & & \\
\hline \multirow[t]{6}{*}{$08 / 10 / 01$} & $B$ & 8 & no & $05 / 12 / 03$ & $J$ & 19 & no \\
\hline & V & 13 & no & & $H$ & 19 & no \\
\hline & $R$ & 14 & no & & & & \\
\hline & $I$ & 7 & no & & & & \\
\hline & $J$ & 26 & no & & & & \\
\hline & $H$ & 26 & no & & & & \\
\hline \multirow[t]{6}{*}{$08 / 11 / 01$} & $B$ & 5 & no & $05 / 14 / 03$ & $J$ & 10 & no \\
\hline & V & 24 & no & & $H$ & 10 & no \\
\hline & $R$ & 23 & no & & & & \\
\hline & $I$ & 5 & no & & & & \\
\hline & $J$ & 24 & no & & & & \\
\hline & $H$ & 24 & no & & & & \\
\hline
\end{tabular}

dos Dias Observatory (Brazópolis, Brazil) ${ }^{1}$ were used simultaneously. The instrumentation was chosen to obtain similar fields of view and time resolution in the optical and near-infrared observations. Additionally, some data were obtained in July 2002 and May 2003, but only in the near-infrared bands. The details of the observational program are described below and the log of the observations can be seen in Tables 1 and 2 for PKS 2005-489 and PKS 2155-304, respectively.

\subsection{Observations and data-reduction procedures in the optical bands}

For the optical observations, we used the LNA $1.6 \mathrm{~m}$ telescope, working with a CCD of $1024 \times 1024$ pixels with $24 \mu \mathrm{m} /$ pixel and a focal reducer that provided a field of view of $10^{\prime} \times 10^{\prime}$ $\left(0.67^{\prime \prime} /\right.$ pixel $)$. We took frames in the filters $B, V, R$, and $I$ with integration times between 180 and $250 \mathrm{~s}$. Two $V$ and $R$ frames were taken for each $B$ and $I$ observation, except in the observations of PKS 2005-489 in the first two nights, in which the observation in the four bands have the same time sampling.

The frames were trimmed, debiased, and divided by the flat dome as usual. Data reduction for light variability analysis was made with the IRAF package ${ }^{2}$, using the APPHOT task for aperture photometry, with a median model to discount the sky contribution and an aperture of three or four times the FWHM. The

1 Operated by the Laboratório Nacional de Astrofísica (LNA/CNPq).

${ }^{2}$ IRAF is distributed by the National Optical Observatories, which is operated by the Association of Universities for research in Astronomy, Inc., under co-operative agreement with the National Science Foundation. 
Table 2. Log of the observations for PKS 2155-304.

\begin{tabular}{|c|c|c|c|c|c|c|c|}
\hline Date & Filter & $N$ & microvar? & Date & Filter & $N$ & microvar? \\
\hline \multirow[t]{6}{*}{$\overline{07 / 16 / 01}$} & $B$ & 5 & yes & $11 / 10 / 01$ & $B$ & 2 & no \\
\hline & $V$ & 13 & yes & & V & 4 & yes \\
\hline & $R$ & 12 & yes & & $R$ & 4 & yes \\
\hline & $I$ & 6 & yes & & $I$ & 1 & no \\
\hline & $J$ & 22 & no & & $J$ & 6 & no \\
\hline & $H$ & 23 & no & & $H$ & 6 & no \\
\hline \multirow[t]{6}{*}{$07 / 17 / 01$} & $B$ & 7 & no & $07 / 08 / 02$ & $J$ & 6 & no \\
\hline & V & 12 & no & & $H$ & 7 & no \\
\hline & $R$ & 11 & no & & & & \\
\hline & $I$ & 7 & no & & & & \\
\hline & $J$ & 29 & no & & & & \\
\hline & $H$ & 28 & no & & & & \\
\hline \multirow[t]{6}{*}{$07 / 18 / 01$} & $\bar{B}$ & 13 & yes & $07 / 10 / 02$ & $\bar{J}$ & 5 & no \\
\hline & V & 24 & yes & & $H$ & 5 & no \\
\hline & $R$ & 22 & yes & & & & \\
\hline & $I$ & 12 & yes & & & & \\
\hline & $J$ & 25 & no & & & & \\
\hline & $H$ & 27 & no & & & & \\
\hline \multirow[t]{6}{*}{$08 / 12 / 01$} & $B$ & 10 & no & $05 / 14 / 03$ & $J$ & 22 & no \\
\hline & V & 41 & yes & & $H$ & 21 & no \\
\hline & $R$ & 39 & yes & & & & \\
\hline & $I$ & 8 & yes & & & & \\
\hline & $J$ & 54 & no & & & & \\
\hline & $H$ & 54 & no & & & & \\
\hline $\begin{array}{l}11 / 08 / 01 \\
\end{array}$ & $J$ & 2 & no & & & & \\
\hline
\end{tabular}

chosen apertures followed the recommendations of Cellone et al. (2000), to insure that the resulting light curves are free of the influence of the host galaxies, whose contribution can change due to seeing fluctuations.

\subsection{Near-infrared observations and data reduction procedures}

The data in the near-infrared range were taken with the Boller \& Chivens $0.6 \mathrm{~m}$ telescope at the LNA, using an infrared camera (CamIV) equipped with a HAWAII $1024 \times 1024$ pixels $\mathrm{HgCdTe}$ detector $(18.5 \mu \mathrm{m} / \mathrm{pixel})$. The field of view of the resulting frames has $8^{\prime} \times 8^{\prime}$, with $0.47^{\prime \prime} /$ pixel. The observations were made with the $J$ and $H$ filters.

For each filter two frames were taken, with a two arcmin offset between them, to discount the sky and background contribution. The integration time for each frame was $60 \mathrm{~s}$, resulting in a time resolution of about $6 \mathrm{~min}$. The reduction was made with the IRAF tasks developed for the CamIV data analysis by F. Jablonski ${ }^{3}$. The frames were corrected by flat field, dark count contribution, and a mask for bad pixels in the detector was applied. After the combination of the two offset frames, the instrumental magnitudes were calculated with APPHOT task. As in the optical observations, we used an aperture that was three or four times the FWHM to insure that the host-galaxy contribution is completely included and does not change due to the variable seeing.

\section{The resulting light curves}

The light curves were constructed in differential mode. For this purpose, we selected one reference star to transform counts to flux density. A set of comparison stars were also selected to

\footnotetext{
${ }^{3}$ http://www. Ina.br/instrum/Camiv/scripts.html
}

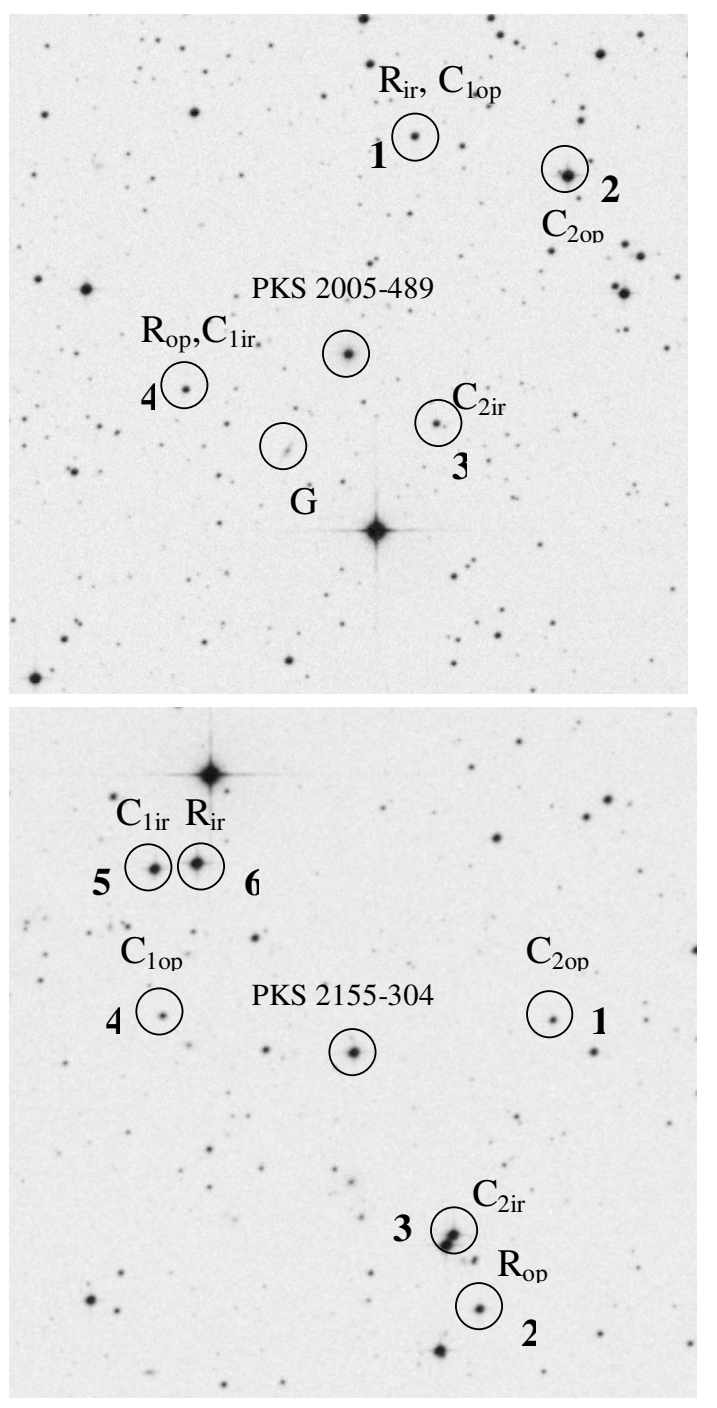

Fig. 1. DSS field image of PKS 2005-489 (top) and PKS 2155-304 (bottom) $\left(B\right.$ band, field of view of $\left.10.5^{\prime} \times 10.5^{\prime}\right)$. The stars used as reference and control in the optical and infrared observations are indicated, along with the galaxy used to study the influence of host contribution in the PKS 2005-489 light curve (see text).

construct the control light curves. Comparison and reference stars chosen in the source field are indicated in the $\mathrm{DSS}^{4}$ images presented in Fig. 1. The selected reference stars are as bright as the blazar or a little fainter, following the recommendations in Romero et al. (2002), and were chosen among those that presented small fluctuations in their light curves. Comparison and reference stars constitute a well-distributed set of objects in the field, some of them common to other variability studies and to field analysis (Hamuy \& Maza 1989; Romero et al. 1999; Rector \& Perlman 2003). However, we did not find any calibrated star in the field of view for the $I$ band, so we observed standard stars from Graham (1982) for calibration purposes. The resulting calibrated magnitudes for the comparison stars in the $I$ band are (following the nomeclature in the Fig. 1): for PKS 2005-489 $m_{1}=13.35 \pm 0.17, m_{2}=14.30 \pm 0.06$, $m_{4}=13.73 \pm 0.08$; for PKS 2155-304 $m_{1}=14.03 \pm 0.08$, $m_{2}=13.32 \pm 0.07, m_{4}=14.55 \pm 0.09$.

We did not necessarily use the same set of stars for the optical and infrared observations, since only a few objects are strong

\footnotetext{
${ }^{4}$ http://archive.stsci.edu/dss/index.html
} 
Table 3. Flux contribution from host galaxies of PKS 2005-489 and PKS 2155-304.

\begin{tabular}{lcc}
\hline \hline Filter & $\begin{array}{c}\text { PKS 2005-489 } \\
F(\mathrm{mJy})\end{array}$ & $\begin{array}{c}\text { PKS 2155-304 } \\
F(\mathrm{mJy})\end{array}$ \\
\hline$B$ & 1.5 & 2.5 \\
$V$ & 3.1 & 3.5 \\
$R$ & 4.8 & 5.2 \\
$I$ & 7.6 & 6.5 \\
$J$ & 14.6 & 3.6 \\
$H$ & 22.5 & 12.2 \\
\hline
\end{tabular}

enough in the infrared bands. The adopted error bars in the light curves correspond to the dispersion of the $\mathrm{C} 1$ control star light curve, which in most cases is within the same order of magnitude as the errors obtained directly from the aperture photometry.

The redenning corrections were made using the values for $A_{V}$ given by Bersanelli et al. (1992) and the relation between $A_{V}$ and the absorption in the other bands determined by Cardelli et al. (1989), resulting in $A_{B}=0.44, A_{V}=0.33, A_{R}=0.25$, $A_{I}=0.16, A_{J}=0.09$, and $A_{H}=0.06$ for PKS 2005-489 and $A_{B}=0.15, A_{V}=0.11, A_{R}=0.08, A_{I}=0.05, A_{J}=0.03$, and $A_{H}=0.02$ for PKS 2155-304. The fluxes were calculated in the usual way: $F_{\Delta v}(\mathrm{mJy})=F_{\Delta v 0}(\mathrm{mJy}) 10^{\left(-0.4 \mathrm{~m}_{\Delta v}\right)}$, where $F_{\Delta v 0}$ is the flux at zeropoint for each spectral band ( $\Delta v$, Bessel 1979 for the optical, Campins et al. 1985 for the near-infrared bands).

The contribution of the host galaxies to the observed fluxes can contaminate the light curves, since their angular sizes are smaller than the apertures used in the photometry, as shown by the radial galaxy profiles presented by Urry et al. (2000) and Cheung et al. (2003) for PKS 2005-489, and by Kotilainen \& Ward (1994) for PKS 2155-304. Based on these data, a fixed flux contribution, presented in Table 3, was subtracted from the data at each band. In the case of PKS 2005-489, for which only the $R$ band host magnitude was available, the flux contribution in the other optical bands was derived based on the general color properties of S0 galaxies (Fukugita et al. 1995).

The amplitude of the variations in the observed blazars was estimated using the expression given by Heidt \& Wagner (1996), but applied to flux densities instead of magnitude differences:

$Y(\%)=\frac{100}{\langle D\rangle} \sqrt{\left(D_{\max }-D_{\min }\right)^{2}-2 \sigma_{\mathrm{c}}^{2}}$

where $D_{\max }$ and $D_{\min }$ are the maximum and minimum values of the flux density, respectively, $\langle D\rangle$ is the mean value, and $\sigma_{\mathrm{c}}$ is the dispersion of the control light curve, based on the $\mathrm{C} 1$ star.

The confidence level of the variability is usually quantified by the factor $C=\sigma_{\mathrm{s}} / \sigma_{\mathrm{c}}$, where $\sigma_{\mathrm{s}}$ is the dispersion of the source light curve. The variability detection is considered real when $C>$ 2.576, which corresponds to a confidence of $99 \%$ (Romero et al. 1999). The values of $Y$ and $C$ for each night and filter in which variability was observed can be seen in Table 4.

\subsection{Light curves of PKS 2005-489}

During the nights of 16 and 17 July, in the first campaign, rapid variations were found on time scales of hours at all bands but with smaller statistical significance in the near-infrared, as can be seen in Table 4. The light curves for the six filters for these nights are presented in Fig. 2, together with the control light curves in the $V$ and $J$ bands included as examples.

The flux density increased during the two nights but decreased during the interval between them, at all optical wavelengths except the $V$ band, where the decrease was still present
Table 4. Values of the parameters $Y$ and $C$ for PKS 2005-489 and PKS 2155-304 detected microvariations.

\begin{tabular}{lccccc}
\hline \hline & & PKS & $2005-489$ & PKS & $2155-304$ \\
\hline Date & Filter & $Y(\%)$ & $C$ & $Y(\%)$ & $C$ \\
\hline $07 / 16 / 01$ & $B$ & 9.2 & 6.1 & 4.8 & 12.1 \\
& $V$ & 7.7 & 9.5 & 16.1 & 25.5 \\
& $R$ & 15.1 & 14.4 & 25.7 & 45.4 \\
& $I$ & 4.2 & 4.7 & 2.2 & 6.7 \\
& $J$ & 11.9 & 3.4 & - & - \\
& $H$ & 8.9 & 3.7 & - & - \\
\hline $07 / 17 / 01$ & $B$ & 6.6 & 6.7 & - & - \\
& $V$ & 10.8 & 15.6 & - & - \\
& $R$ & 15.0 & 15.5 & - & - \\
& $I$ & 2.5 & 5.9 & - & - \\
& $J$ & 10.9 & 3.7 & - & - \\
& $H$ & 9.4 & 5.2 & - & - \\
\hline $07 / 18 / 01$ & $B$ & - & - & 2.7 & 5.7 \\
& $V$ & - & - & 6.6 & 12.6 \\
& $R$ & - & - & 30.3 & 15.2 \\
& $I$ & - & - & 1.6 & 3.7 \\
\hline $08 / 12 / 01$ & $B$ & - & - & 15.0 & 7.6 \\
& $V$ & - & - & 10.0 & 14.6 \\
& $R$ & - & - & 13.9 & 8.7 \\
& $I$ & - & - & 14.8 & 12.3 \\
\hline $11 / 10 / 01$ & $V$ & - & - & 9.6 & 47.7 \\
& $R$ & - & - & 13.2 & 85.3 \\
\hline
\end{tabular}
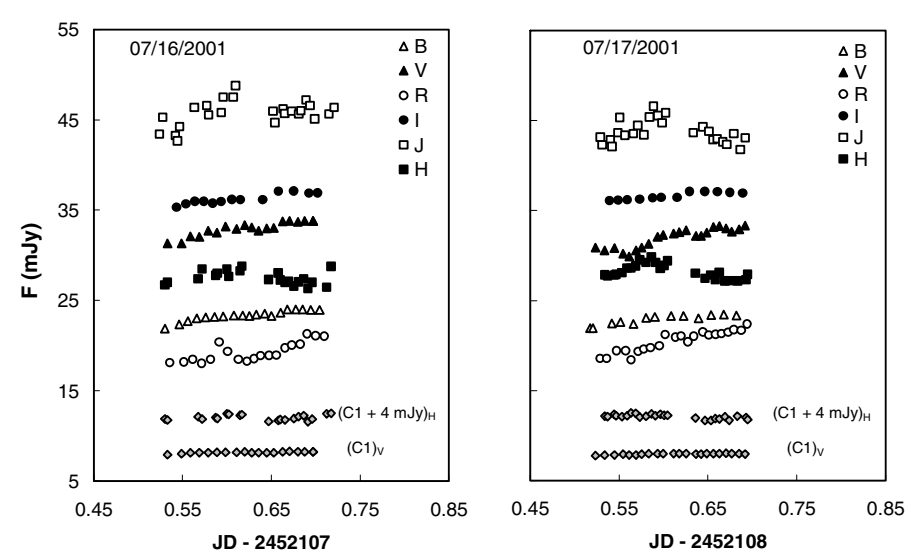

Fig. 2. Light curves of the BL Lac PKS 2005-489 in six bands during the two consecutive nights in July 2001 in which short-term variability was detected. The control light curves for the $V$ and $H$ bands are also shown to exemplify their stability.

at the beginning of the second night. The behavior of the light curves in the infrared wavelengths was similar during the first night, but with smaller amplitude, and during the second night the flux density started to decrease after a few hours of observation. It is interesting to notice that the $I$ band light curve shows a transition between the optical and infrared behavior, pointing out the reliability of the two sets of observations taken with two different telescopes.

To further assert the reliability of our results we present , as an example, the $R$ band light curve of a spiral galaxy in the field of PKS 2005-489 in Fig. 3, indicated by $(G)$ in Fig. 1, during the night of 17 July, when the largest variation amplitude was observed for this BL Lac object. The absence of light variations in the galaxy implies that the variability in PKS 2005-489 is not due to the contribution of low-surface brightness components in the host galaxy (Cellone et al. 2000). Another test is to verify of the behavior of the FWHM (indication of seeing variations) 


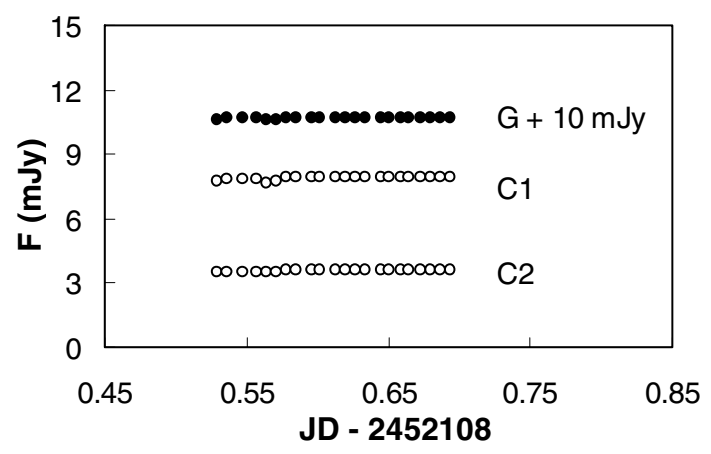

Fig. 3. Light curve of the galaxy indicated in Fig. 1 (G) and the respective control light curves for the night of 07/17/01 in the $R$ band. The absence of variations confirms that the variability detected in PKS 2005-489 light curve was not caused by the influence of the host galaxy.
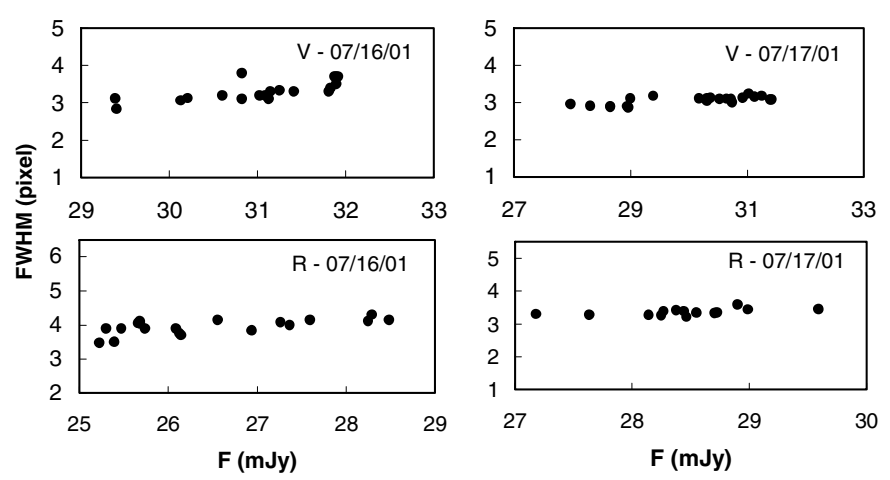

Fig. 4. Comparison between the FWHM evolution and PKS 2005-489 flux density during the nights of July 16 and 17 with the $V$ and $R$ filters.

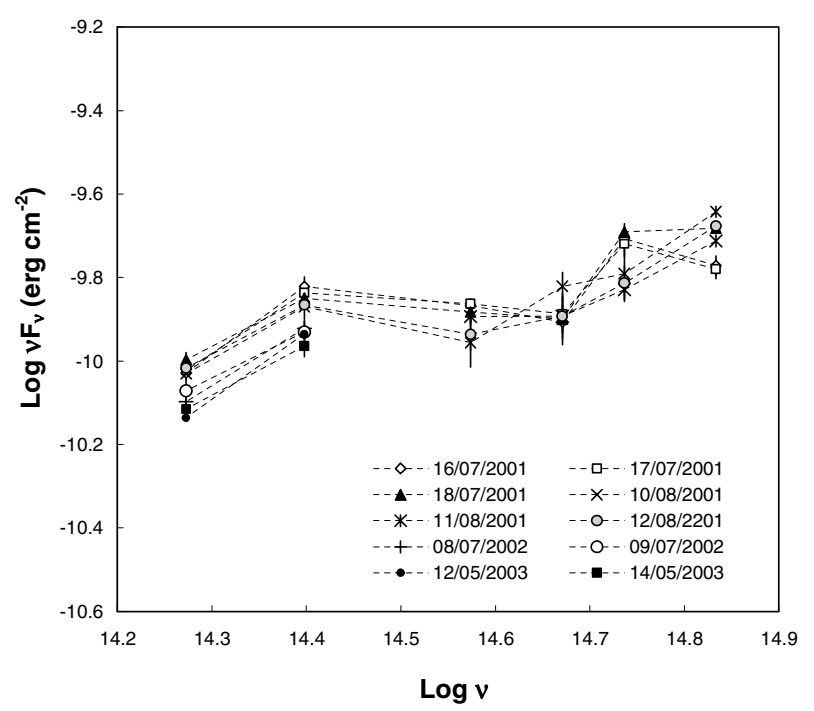

Fig. 5. Spectral energy distribution of PKS 2005-489 from data taken between 2001 and 2003. The error bars correspond to the standard deviation in daily averages.

during the monitoring period (Jang \& Miller 1997). Figure 4 shows that apparently there is no correlation between flux density and FWHM that could explain the variability observed in the light curves. In fact, in the Sperman correlation coefficient $\left(r_{\mathrm{S}}\right)$ gives a value of about 0.65 in all cases, which means no correlation or a weak one.

PKS 2005-489 did not show microvariability during the observations in August 2001. The long-term spectral behavior can
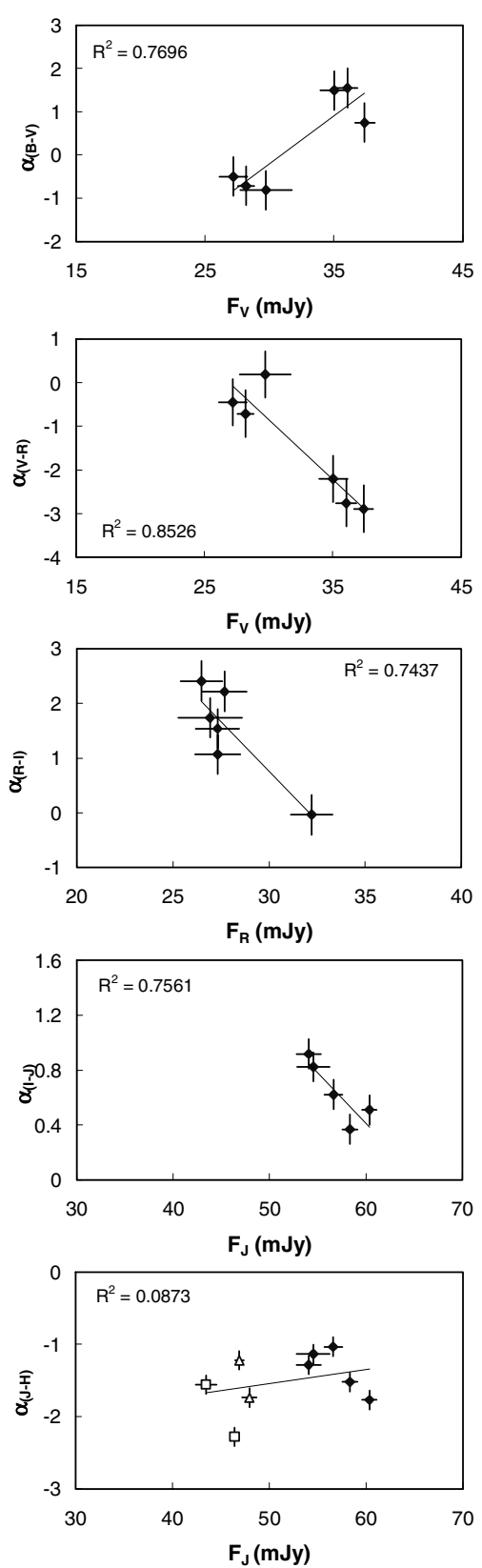

Fig. 6. Correlation between spectral indices calculated between two consecutive bands and the corresponding flux densities in PKS 2005489. The solid lines represent a linear fit to the spectral indices. The goodness of the fit $\left(R^{2}\right)$ is indicated in each plot. In the last panel, the open triangules and the open squares indicate the 2002 and 2003 measurements, respectively.

be seen in Fig. 5, where the additional data taken on July 2002 and May 2003 in the infrared were also included. The points are the average flux density for each night and the error bars are the standard deviation. In the infrared bands, PKS 2005-489 brightness decreased by about $19 \mathrm{mJy}$ and $16 \mathrm{mJy}$ in the the $J$ and $H$ bands, respectively.

As we can see, the spectrum is variable and not smooth between the different wavebands. For that reason we calculated the spectral indices $\left(S(v) \propto v^{-\alpha}\right)$ between each pair of consecutive bands and found them correlated to the corresponding flux densities, as shown in Fig. 6. Only for $\alpha_{(B-V)}$ does the spectral index become harder when the $V$ band flux density increases. In the infrared no correlation seems to exist. 

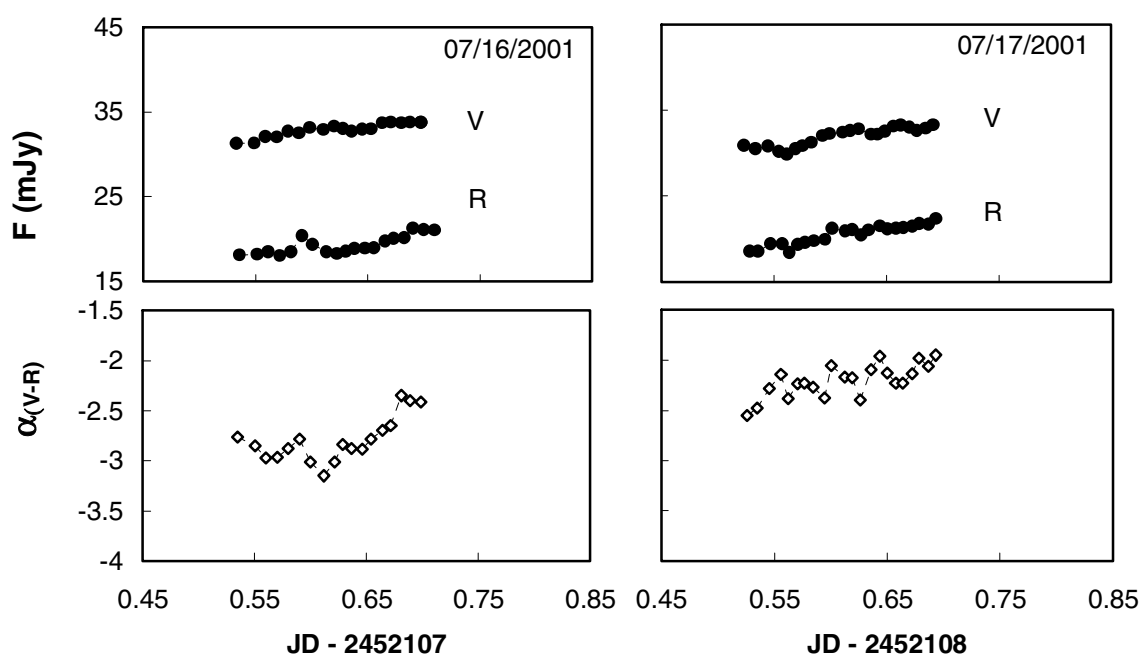

Fig. 7. Light curves of the BL Lac PKS 2005-489 in the $V$ and $R$ bands during the nights of July 16 and 17, 2001, and the spectral indices calculated between these two bands on time scales of a few minutes.
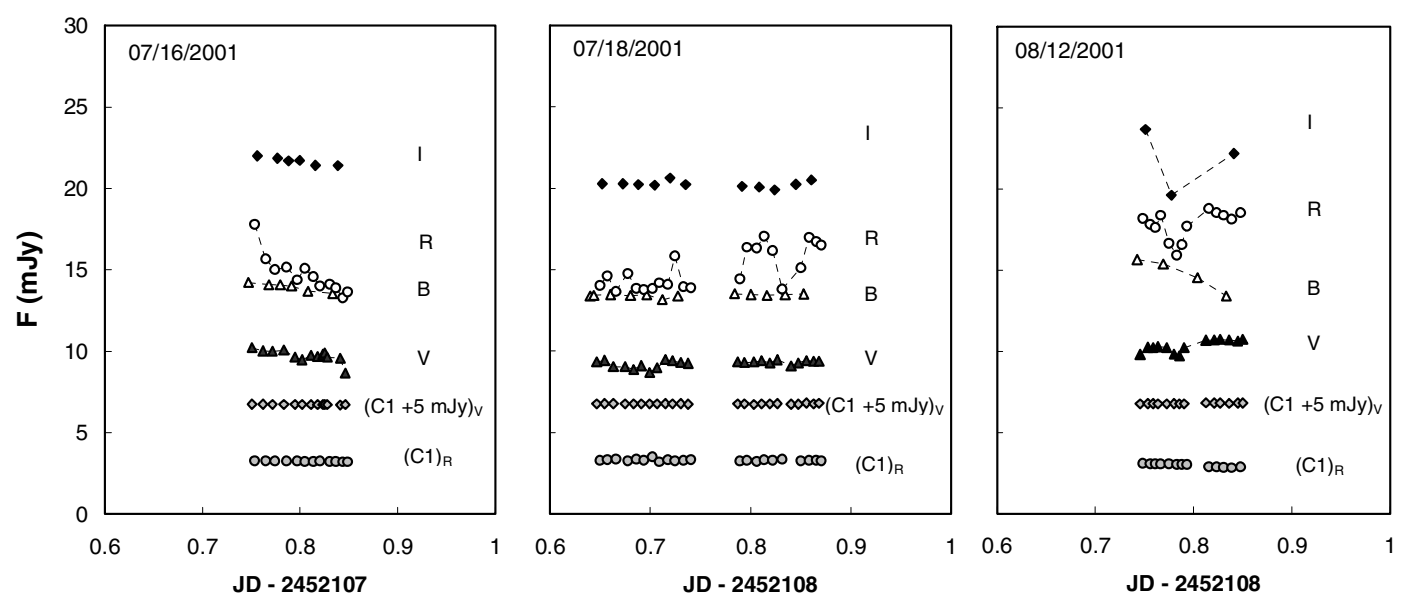

Fig. 8. Light curve of the BL Lac PKS 2155-304 during three nights in July and August 2001 in six bands. The control light curves in the $V$ and $H$ band are also shown to exemplify their stability.

Additionally, by taking advantage of the good data sampling of the $V$ and $R$ light curves obtained during the two nights in which short-term variability was detected, we calculated the instantaneous spectral index between these two filters. The results can be seen in Fig. 7; in general, the spectral index becomes harder as the flux density increases, as observed in other sources (Wagner et al. 1996).

\subsection{Light curves of PKS 2155-304}

Microvariability was detected in PKS 2155-304 during three nights, occurring only in the optical bands, as shown in Fig. 8. In fact, we did not find microvariability in the the near-infrared wavelengths at any time during the monitoring period. The control light curves for the $V$ and $J$ bands are shown as an example to check the accuracy of the observations.

The long-term behavior of the spectral energy distribution can be seen in the Fig. 9. On time scales of months, we observed variations in the six bands between July and November 2001. The amplitude of the variations was different for each band. Additionally, PKS 2155-304 was observed in the infrared in two nights in July 2002 for about one hour and in May 2003 for about three hours. The source presented a brightness decrease of about 14 and $17 \mathrm{mJy}$ in $J$ and $H$, respectively, from November 2001 to May 2003, as also shown in Fig. 9.

The near-infrared observations of PKS 2155-304 in July 2002 and May 2003 were obtained, respectively, a few days and a month before detection of the source at TeV energies, by the HESS collaboration (Aharonian et al. 2005a). The fluxes of the TeV detections were $I(>1 \mathrm{TeV})=(85.1 \pm 16.1) \times$ $10^{-9} \mathrm{~m}^{-2} \mathrm{~s}^{-1}(13 \sigma$ of significance) and $I(>1 \mathrm{TeV})=(9.45 \pm$ $1.27) \times 10^{-9} \mathrm{~m}^{-2} \mathrm{~s}^{-1}(21.1 \sigma$ of significance $)$ in 2002 and 2003 , respectively. In the first epoch the $H$ band flux density was about $20.2 \pm 0.6 \mathrm{mJy}$, while in the second it was $16.0 \pm 0.5 \mathrm{mJy}$.

As for PKS 2005-489, the spectral energy distribution is complex, so we also calculated the spectral indices between each pair of wavebands. The results are shown in Fig. 10, where we also can see a correlation between spectral index and flux density.

\section{Duty cycle}

Using the results of the observational campaign presented here, we can quantify the incidence of microvariability using the definition of a duty cycle given by Romero et al. (1999):

$\mathrm{DC}=100 \frac{\sum_{i=1}^{n} N_{i}\left(1 / \Delta t_{i}\right)}{\sum_{i=1}^{n}\left(1 / \Delta t_{i}\right)} \%$ 


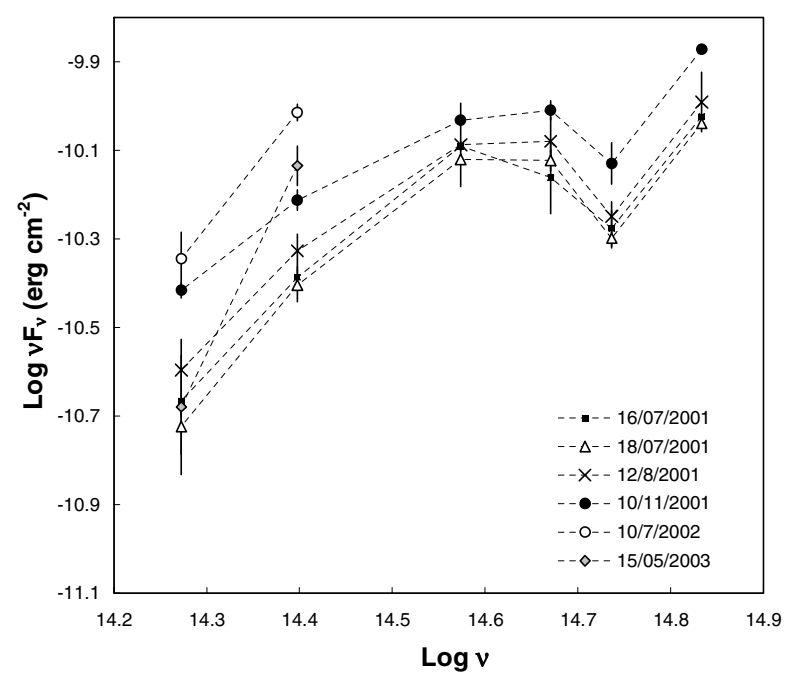

Fig. 9. Spectral energy distribution of PKS 2155-304 between July 2001 and May 2003 in the six filters. We binned the data for better visualization; the error bars correspond to the standard deviation of the daily averages.

where $n$ is the number of nights, $N_{i}$ is equal to 1 or 0 , depending on whether or not variations were detected in the interval $\Delta t_{i}=$ $\Delta t_{i, \text { obs }}(1+z)^{-1}$, measured in rest frame of the source of redshift $z$, while $\Delta t_{i, \text { obs }}$ is the time interval in the observer's rest frame.

We calculated the value of DC both for the optical and near-infrared bands. In the optical wavelengths, the duty cycle was $29.1 \%$ for PKS 2005-489 and 31.2\% for PKS 2155-304 (disregarding the November 2001 night, when only a few observations were available). As mentioned before, although PKS 2005489 was initially found as an RBL, its SED from radio to X-rays suggests that it may be considered an XBL (e.g., Padovani et al. 2001). Therefore, assuming both PKS 2005-489 and PKS 2155304 belong to the XBL category, we found a total duty cycle of $30.6 \%$. These results agree with the $27.9 \%$ obtained by Romero et al. (1999) for a sample of three XBLs observed at the same spectral bands. The duty cycle for microvariability in the near-infrared, calculated for the first time based on the results of PKS 2005-489 monitoring, resulted in 31.6\%.

\section{Conclusions}

In this work we present the results of a monitoring program of the BL Lacs PKS 2005-489 and PKS 2155-304 in six spectral bands $(B, V, R, I, J$, and $H)$, covering time scales ranging from a few minutes to some years. For both sources the contribution of the host galaxy was subtracted from the observed flux after the interstellar reddening correction was applied.

PKS 2005-489 was observed during three consecutive nights in July and August 2001, with an average sample of 20 observations per night in all bands except for $B$ and $I$, where only half of them were taken. It was also observed two nights in the the $J$ and $H$ bands in July 2002 and May 2003. Short time-scale variability was observed during two nights in July 2001; this is the first time in which variability in time scales of a few minutes was detected in the six bands. The confidence of the detection was checked through the analysis of the atmospheric influence and the adequate choice of the aperture for the photometric measurements. Long-term near-infrared variations were also found, with a brightness decrease between July 2001 and May 2003.
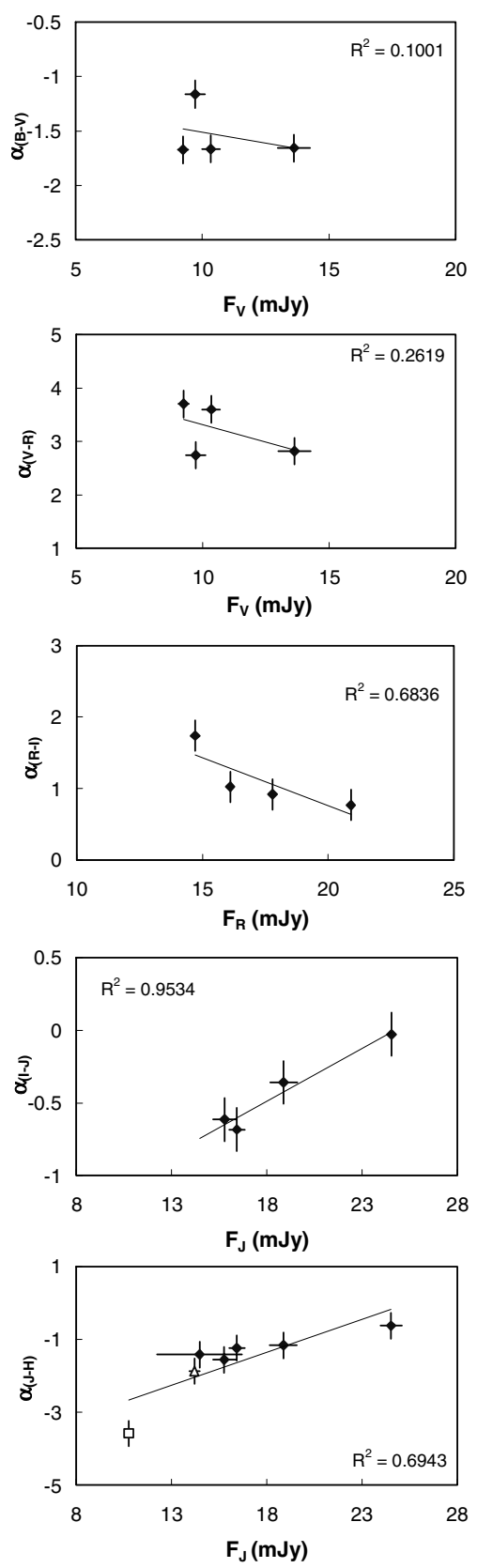

Fig. 10. Correlation between spectral indices calculated between two consecutive bands and the corresponding flux densities in PKS 2155304. The solid lines represent a linear fit to the spectral indices. The goodness of the fit $\left(R^{2}\right)$ is indicated in each plot. In the last panel, the open triangle and the open square indicate the 2002 and 2003 measurements, respectively.

PKS 2155-304 was observed in the six bands during three consecutive nights in July 2001, one night in August 2001, and two nights in November 2001. It was also observed in the infrared $J$ and $H$ bands during two nights in July 2002 and one night in May 2003. We detected brightness variability in all bands on long time-scales but micovariability only at optical bands and at isolated epochs, as it is an already known characteristic of this object. The near-infrared observations of PKS 2155304 in July 2002 and May 2003 were obtained, respectively, a few days and a month before detection of the source at $\mathrm{TeV}$ energies, by the HESS telescopes (Aharonian et al. 2005a).

Considering both sources, the optical duty cycle for microvariability was found to be $30.6 \%$, similar to what is reported 
by Romero et al. (1999) for this class of sources (27.9\%). For the first time the near-infrared duty cycle for microvariability was found to be $31.6 \%$, based on PKS 2005-489 monitoring.

A complex spectral energy distribution between optical and infrared wavelengths was found for both objects. Similar behavior in the infrared was found by Riffel et al. (2006) in a large sample of quasars and Seyfert 1 galaxies. For this reason, spectral indices between two consecutive bands were calculated using daily flux averages, and a correlation between them and the corresponding flux density was found for most of the cases. For PKS 2005-489, intranight spectral indices between $V$ and $R$ were obtained for the two nights in which variability was detected, thanks to the good sampling of the light curves in those occasions. On short time-scales, a correlation was also found in this source, with the spectrum becoming harder when the flux increased.

In a first analysis, the microlensing scenario can be discarded to explain the observed behavior in the light curves, because the variations were not achromatic. Eclipses, as well as intrinsic mechanisms like the evolution of relativistic jets, are possible explanations, if we consider that the variability occurs in a region with a spectral index different from that of the quiescent structure. Between these two mechanisms, jets seem to be better located to produce of the observed variability in the two objects, because of the short time scales involved and the changes in the spectral shape. In both cases, the spectral evolution is not smooth, presenting bumps in the optical range and a spectral break between optical and near-infrared energies. This complex behavior can be explained by the existence of shortlived components, such as shocks, superposed to the quiescent jet contribution.

More constraints on the possible physical scenarios capable of explaining the occurrence of rapid variations in these objects must be obtained with further intensive monitoring campaigns covering spectral regions for which we could obtain similar time resolution in the light curves.

Acknowledgements. We are grateful to the referee for the comments that helped us to improve significantly this paper. We would like to acknowledge the financial support by FAPESP (São Paulo, Brazil), through the grant 02/13790-5, and $\mathrm{CNPq}$.

\section{References}

Abraham, Z. 2000, A\&A, 355, 915

Aharonian, F., Akhperjanian, A. G., Aye, K.-M., et al. 2005a, A\&A, 430, 865 Aharonian, F., Akhperjanian, A. G., Aye, K.-M., et al. 2005b, A\&A, 436, 17 Andruchow, I., Cellone, S. A., Romero, G. E., Dominici, T. P., \& Abraham, Z. 2003, A\&A, 409, 857

Bersanelli, M., Bouchet, P., Falomo, R., \& Tanzi, E. G. 1992, AJ, 104, 28
Bessel, M. S. 1979, PASP, 91, 589

Böttcher, M., Harvey, J., Joshi, M., et al. 2005, ApJ, 631, 169

Brindle, C., Hough, J. H., Bailey, J. A., Axon, D. J., \& Hyland, A. R. 1986, MNRAS, 221, 739

Campins, H., Rieke, G. H., \& Lebofsky, M. J. 1985, AJ, 90, 896

Cardelli, J. A., Clayton, G. C., \& Mathis, J. S. 1989, ApJ, 345, 245

Cellone, S. A., Romero, G. E., \& Combi, J. A. 2000, AJ, 119, 1534

Clements, S. D., Jenks, A. \& Torres, Y. 2003, AJ, 126, 37

Chadwick, P. M., Lyons, K., McComb, T. J. L., et al. 1999, ApJ, 513, 161

Cheung, C. C., Urry, C. M., Scarpa, R., \& Giavalisco, M. 2003, ApJ, 599, 155

Courvoisier, T. J.-L., Robson, E. I., Hughes, D. H., Blecha, A., \& Bouchet, P. 1988, Nature, 335, 330

Courvoisier, T. J.-L., Blecha, A., Bouchet, P., et al. 1995, ApJ, 438, 108

Dominici, T. P., Abraham, Z., Teixeira, R., \& Benevides-Soares, P. 2004, AJ, 128,47

Edelson, R., Krolik, J., Madjeski, G., et al. 1995, ApJ, 438, 120

Falomo, R., Giraud, E., Maraschi, L., Tanzi, E. G., \& Treves, A. 1991, ApJ, 380, 67

Fukugita, M., Shimasaku, K., \& Ichikawa, T. 1995, PASP, 107, 945

Graham, J. A. 1982, PASP, 94, 244

Ghosh, K. K., Ramsey, B. D., Sadun, A. C., \& Soundararajaperumal, S. 2000, ApJS, 127, 11

Gu, M. F., Lee, C.-U., Pak, S., Yim, H. S., \& Fletcher, A. B. 2006, A\&A, 450, 39

Hamuy, M., \& Maza, J. 1989, AJ, 97, 720

Heidt, J., \& Wagner, S. 1996, A\&A, 305, 42

Heidt, J., Wagner, S., \& Wilhelm-Erkens, U. 1997, A\&A, 325, 27

Jang, M., \& Miller, R. H. 1995, ApJ, 452, 582

Jang, M., \& Miller, R. H. 1997, AJ, 114, 565

Kraus, A., Quirrenbach, A., Lobanov, A. P., et al. 1999, A\&A, 344, 807

Kotilainen, J. K., \& Ward, M. J. 1994, MNRAS, 266, 953

Kotilainen, J. K., Falomo, R., \& Scarpa, R. 1998, ApJ, 336, 479

Lorenzetti, D., Massaro, E., Perola, G. C., \& Spnoglio, L. 1989, ApJS, 71, 175

Marscher, A. P., Gear, W. K., \& Travis, J. P. 1992, in Variability of blazars, ed. E. Valtaoja, \& M. Valtonen (Cambridge University Press), 85

Miller, H. R., \& McAlister, H. A. 1983, ApJ, 272, 26

Paltani, S., Courvoisier, T. J.-L., Blecha, A., \& Bratschi, P. 1997, A\&A, 327, 539

Padovani, P., Costamante, L., Giommi, P., et al. 2001, MNRAS, 328, 931

Pesce, J. E., Urry, C. M., Maraschi, L., et al. 1997, ApJ, 486, 770

Pian, E., Urry, C. M., Treves, A., et al. 1997, ApJ, 486, 784

Qian, S. J., Quirrenbach, A., Witzel, A., et al. 1991, A\&A, 241, 15

Raiteri, C. M., Villata, M., Tosti, G., et al. 2003, A\&A, 402, 151

Raiteri, C. M., Villata, M., Ibrahimov, M. A., et al. 2005, A\&A, 438, 39

Rector, T. A., \& Perlman, E. S. 2003, AJ, 126, 47

Riffel, R., Rodriguez-Ardila, A., \& Pastoriza, M. G. 2006, A\&A, 457, 61

Romero, G. E., Surpi, G., \& Vucetich, H. 1995, A\&A, 301, 641

Romero, G. E., Cellone, S. A., \& Combi, J. A. 1999, A\&AS, 135, 477

Romero, G. E., Cellone, S. A., \& Combi, J. A. 2000, AJ, 120, 1192

Romero, G. E., Cellone, S. A., Combi, J. A., \& Andruchow, I. 2002, A\&A, 390, 431

Urry, C. M., Treves, A., Maraschi, L., et al. 1997, ApJ, 486, 799

Urry, C. M., Scarpa, R., O’Dowd, M., et al. 2000, ApJ, 532, 816

Valtaoja, L., Valtaoja, E., Shakhovsky, N. M., Efimov, Yu. S., \& Silampää, A. 1991, AJ, 101, 78

Véron-Cetty, M. P., \& Véron, P. 1998, A catalogue of Quasars and Active Galactic Nuclei (8o edition), ESO Scientific Report, 18

Wagner, S., \& Witzel, A. 1995, ARA\&A, 33, 163

Wagner, S., Witzel, A., Heidt, J., et al. 1996, AJ, 111, 2187

Whiting, M., Oshlack, A., \& Webster, R. 2002, PASA, 19, 222 